\title{
Vaginal leiomyoma, post hysterectomy, mimicking vault prolapse: case report
}

\author{
Jharna Behura $^{1 *}$, Poonam Bagga ${ }^{1}$, Anjali Mathur ${ }^{2}$ \\ ${ }^{1}$ Department of Obstetrics and Gynecology, ${ }^{2}$ Department of Pathology, Kasturba Hospital, Delhi, India
}

Received: 30 August 2020

Accepted: 06 October 2020

\section{*Correspondence:}

Dr. Jharna Behura,

E-mail: jharnabehura@yahoo.co.in

Copyright: $\odot$ the author(s), publisher and licensee Medip Academy. This is an open-access article distributed under the terms of the Creative Commons Attribution Non-Commercial License, which permits unrestricted non-commercial use, distribution, and reproduction in any medium, provided the original work is properly cited.

\begin{abstract}
Leiomyomas are common benign smooth muscle tumours of the uterus. Leiomyoma of the vagina are very rare with only about 300 reported cases. Correct diagnosis and differentiation from urethral lesions are important but is usually difficult on clinical examination alone. A 40-year P3L3, with a previous history of abdominal hysterectomy 5 years back, for multiple fibroids presented with complaints of a mass descending per-vagina since the last 2 years. Since the last 6 months she had dyspareunia, dysuria and perineal discomfort. On external appearance the mass looked like a vault prolapse. However, on vaginal examination, it appeared as a single well circumscribed mass arising from the anterior vaginal wall and the vault. Transvaginal sonography showed a hypoechoic mass arising from the anterior vaginal wall of dimension $50 \times 54 \times 63 \mathrm{~mm}$ pressing on the urethra. Contrast CT revealed a rounded homogeneous welldefined soft tissue density mass lesion measuring approximately $63 \times 52 \mathrm{~mm}$ in the region of the vaginal vault interposed between the bladder base and the rectum. Bilateral ovaries and adnexa appeared normal. Surgical excision of the tumour through the vagina was done. Histopathology confirmed leiomyoma. The diagnosis of vaginal leiomyoma is based on careful examination and preoperative imaging both by ultrasonography and a CT scan/MRI for ascertaining its location, extent, its relationship to adjacent structures and characterization before attempting surgical excision. The correct clinical diagnosis of a vaginal fibroid is frequently overlooked in favour of much more common urethral diverticulum.
\end{abstract}

Keywords: Vaginal leiomyoma, Ultrasonography, CT Scan, Magnetic resonance imaging, Surgical excision

\section{INTRODUCTION}

Leiomyoma of the vagina are rare benign smooth muscle tumours, with only about 300 cases being reported in literature. ${ }^{1}$ These tumours mostly arise from the anterior vaginal wall. They present with varied clinical symptoms like dyspareunia, dysuria, pain and bleeding per vaginum, depending on the size and location. ${ }^{2}$ Vaginal leiomyomas may sometimes occur concurrently with leiomyomas elsewhere in the body, particularly the uterus and cervix. ${ }^{3}$ A high index of suspicion is required for a clinical diagnosis of vaginal leiomyoma because the tumour could be easily mistaken for a cystocele, urethrocele, abscess of skene's duct, urethral diverticulum, vaginal cyst, Gartner duct cyst and Bartholin gland cyst or vaginal malignancy. ${ }^{4,5}$ Correct diagnosis and differentiation from urethral lesions may be difficult when based on clinical examination alone. MRI/CT scan helps in accurate localisation, estimates the extent and characterization of the mass and ascertains its relationship to adjacent structures. ${ }^{6}$ Surgical enucleation is the treatment of choice. Recurrences though uncommon are reported. The definite diagnosis is made on histological findings consistent with a mix of smooth muscle and fibrous stroma.

The authors are reporting a case of vaginal leiomyoma that appeared like vault prolapse in a woman who had undergone hysterectomy for multiple fibroids few years back. 


\section{CASE REPORT}

A 40-year-old lady P3L3 presented to our Gynae OPD with complaints of something descending per-vaginum since the last 2 years, more during squatting, coughing and sneezing. The mass was initially small and had gradually increased to its present size. She also complained of dyspareunia, dysuria, perineal discomfort and vaginal bleeding off and on, for the last 6 months. She had a history of total abdominal hysterectomy, 5 years back for multiple uterine fibroids. She had no other surgical or medical history. On physical examination, she looked well; her blood pressure was $126 / 84 \mathrm{mmHg}$, pulse was 86 beats/min. and temperature of $37.4^{\circ} \mathrm{C}$.

On external examination a mass was seen coming out of the vagina and appearing as vault prolapse. Per Speculum examination revealed a round solid mass of about $6 \mathrm{~cm} \mathrm{x}$ $5 \mathrm{~cm}$ involving the anterior vagina. The posterior vaginal wall was free. Per vaginal examination revealed a midline mass arising from the anterior vaginal wall and vault, firm in consistency. The surface of the tumour was covered by vaginal epithelium (Figure 1).

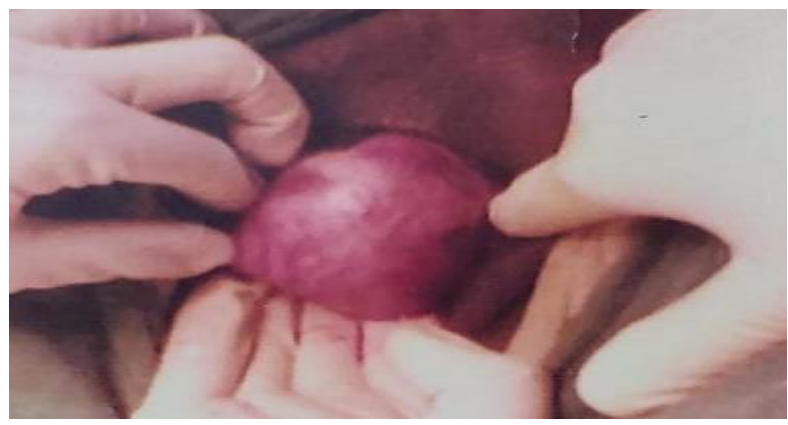

Figure 1: Mass arising from anterior vaginal wall.

Transvaginal sonography showed a hypoechoic mass arising from the anterior vaginal wall of dimension $50 \times 54 \times 63 \mathrm{~mm}$ pressing on the urethra. Urinary bladder appeared normal. Both ovaries appeared normal. Both kidneys appeared normal.

Contrast CT was done which reported, uterus not visualized, likely postoperative status. There is a rounded homogeneous well-defined soft tissue density mass lesion measuring approximately $63 \mathrm{~mm}$ in the region of the vaginal vault interposed between the bladder base and the rectum. Bilateral ovaries and adnexa appear normal (Figure 2).

The location and the image findings supported the diagnosis of a benign vaginal tumour. A decision was taken to surgically excise the mass through the vaginal route. A midline incision was given on the anterior vaginal wall with a Foleys catheter in situ and the mass was easily enucleated from the anterior wall. The vagina was repaired by the two-layer procedure. (Figure 3).
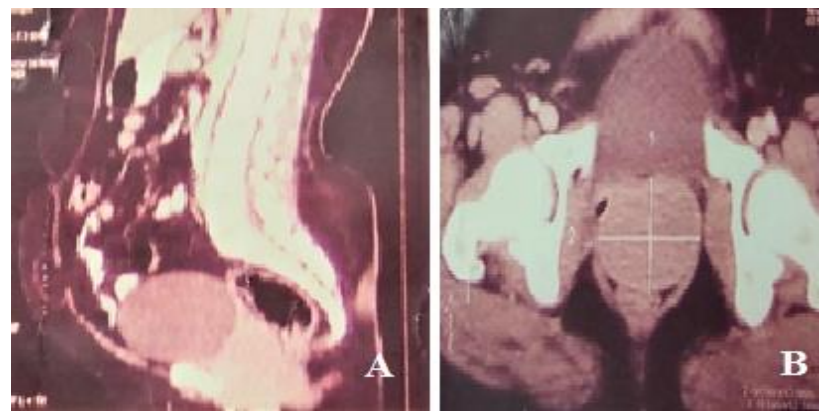

Figure 2: CT scan (A) Contrast CT Anterior posterior view (B) Transverse section, rounded homogeneous well-defined soft tissue density mass lesion $63 \times 52 \mathrm{~mm}$ in the region of the vaginal vault between the bladder base and the rectum.
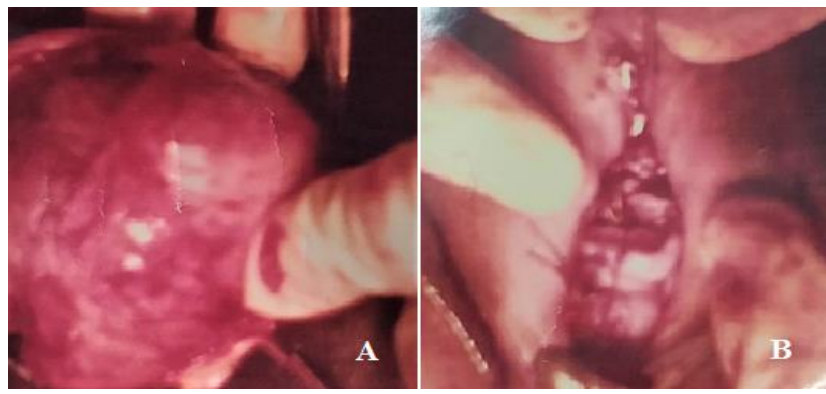

Figure 3: (A) Tumour enucleated (B) Anterior vaginal wall after tumour enucleation.

Gross examination showed a globular mass of $6 \mathrm{cmx} 5 \mathrm{~cm}$ in dimension with a white capsule. Cut section showed a fibrous whorled appearance. Histopathological examination confirmed the diagnosis of a benign leiomyoma of the vagina (Figure 4).

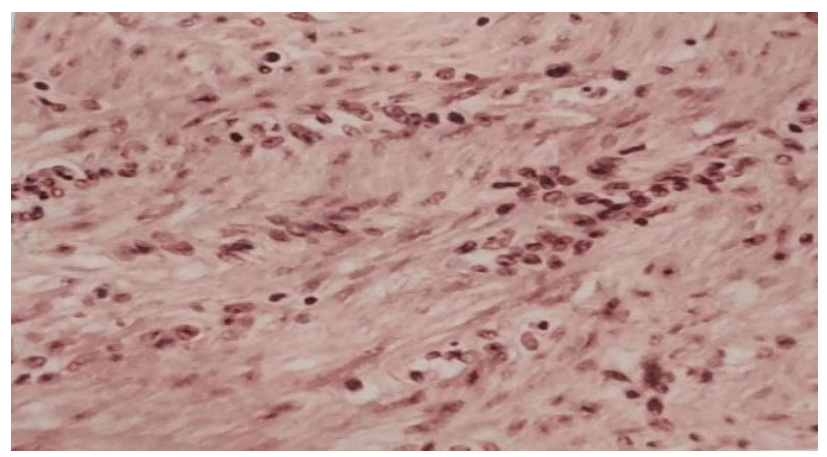

Figure 4: Histopathological appearance confirming leiomyoma.

\section{DISCUSSION}

Leiomyomas are benign tumours of the female genital tract occurring more commonly in the uterus, to some extent in the cervix, less commonly in the round ligament, uterosacral ligament, ovary and inguinal canal. ${ }^{7}$ Leiomyoma of the vagina are rare and until now less than 400 cases have been reported in publications worldwide. 
Vaginal leiomyoma was initially reported in 1733 by Denys de Leyden. ${ }^{8}$ The prevalence of these tumours is estimated to be approx. 1 per 15,000 on the basis of autopsy series reviewed at John Hopkins Hospital. The tumours are common between 35 to 50 years. These tumours characteristically occur as a single well circumscribed mass arising from the midline of the anterior vaginal wall and less commonly from lateral wall and rarely from the posterior vaginal wall. Proposed site of origin includes vaginal smooth muscle, local arterial musculature or smooth muscle of urethra. ${ }^{9,10}$ Leiomyomas of the vagina are usually asymptomatic but depending on their size may present with lower abdominal pain, dyspareunia, dysuria and vaginal bleeding. Careful examination and preoperative imaging are very important. Several entities must be considered in the differential diagnosis of mass localised between vagina and urethra. The correct clinical diagnosis of a vaginal fibroid is frequently overlooked in favour of much more common urethral diverticulum.

Radiologic analysis by ultrasound, CT scan or MRI is mandatory for evaluating a vaginal mass before attempting excision. It helps to ascertain its location, extent, its relationship to adjacent structures and characterization. ${ }^{6}$ Vaginal leiomyoma's are usually single benign and slow-growing tumours but sarcomatous transformation have been reported in literature. MRI particularly plays a pivotal role, when vaginal fibroids grow rapidly, have poor delineation on ultrasound and when there is a suspicion of malignancy.

The treatment is surgical and vaginal approach is recommended. Determination of the primary organ involved and a benign or malignant nature of the mass determines the choice of surgical approach When tumour is large, the combined abdominal and perineal approach can be used. ${ }^{9}$ It is important that the tumour be removed intact and the patient be followed up long term, as recurrences, though rare have been reported. ${ }^{11}$ Histopathologically, it is difficult to distinguish between benign and malignant smooth muscle tumours of the vagina. Tavassoli and Norris established diagnostic criteria where tumours greater than 5 mitoses per high power field and moderate to marked atypism should be considered as leiomyosarcoma. ${ }^{12}$ When the morphological diagnosis is difficult biomarkers like p16, p53 and ki-67 have been used to predict the tumour behaviour. $^{13}$

\section{CONCLUSION}

Vaginal leiomyomas are a rare occurrence. Before a surgical intervention is attempted, evaluation of the vaginal mass should be done by ultrasound and MRI/CT scan to know the extent, characterization and involvement of the mass. They are usually benign in nature but sarcomas have been reported. In large tumours a frozen section is recommended to exclude leiomyosarcoma.

\section{Funding: No funding sources Conflict of interest: None declared \\ Ethical approval: Not required}

\section{REFERENCES}

1. Young SB, Rose PG, Reuter KL. Vaginal fibromyomata: two cases with preoperative assessment, resection, and reconstruction. Obstet Gynecol. 1991;78(5 Pt 2):972-4.

2. Costantini E, Cochetti G, Porena M. Vaginal Paraurethral myxoid leiomyoma: case report and review of the literature. Int Urogynecology J. 2008; 19:1183-5

3. Chakrabarti I, De A, Pati S. Vaginal leiomyoma. J Lif Heal. 2011;2:42-3.

4. Sim CH, Lee JH, Kwak JS, Song SH. Necrotizing ruptured vaginal leiomyoma mimicking a malignant neoplasm. Obstet Gynecol Sci. 2014;57:560-3.

5. Wu Y, Wang W, Sheng X, Kong L, Qi J. A misdiagnosed vaginal leiomyoma: case report. Urol Case Rep. 2015;3:82-3.

6. Sherer DM, Cheung W, Gorelick C, Lee Y-C, Serur E, Zinn HL, et al. Sonographic and magnetic resonance imaging findings of an isolated vaginal leiomyoma. J Ultrasound Med Off J Am Inst Ultrasou Med. 2007;26:1453.

7. Laughlin SK, Stewart EA. Uterine leiomyomas: individualising the approach to a heterogeneous condition. Obstet Gynecol. 2011;117(2 Pt 1):396

8. Young SB, Rose PG, Reuter KL. Vaginal fibromyomata: two cases with preoperative assessment, resection, and reconstruction. Obstet Gynecol. 1991;78(5 Pt 2):972-4.

9. Gowri R, Soundararaghavan S, Oumachigui A, Sistla $\mathrm{SC}$, Iyengar KR. Leiomyoma of the vagina: an unusual presentation. J Obstet Gynaecol Resear. 2003;29(6):395-8.

10. Kaufman RH, Gardner HL. Tumors of the vulva and vagina. Benign mesodermal tumors. Clinic Obstet Gynecol. 1965;8(4):953-81.

11. Dhaliwal LK, Das I, Gopalan S. Recurrent leiomyoma of the vagina. Int $\mathrm{J}$ Gynecol Obstet. 1992;37(4):281-3.

12. Tavassoli FA, Norris HJ. Smooth muscle tumors of the vagina. Obstet Gynecol. 1979;53(6):689-93.

13. Lee CH, Turbin DA, Sung YV, Espinosa I, Montgomery K, Van De Rijn M, et al. A panel of antibodies to determine site of origin and malignancy in smooth muscle tumors. Mode Pathol. 2009;22(12):1519-31.

Cite this article as: Behura J, Bagga $\mathrm{P}$, Mathur A. Vaginal leiomyoma, post hysterectomy, mimicking vault prolapse: case report. Int J Reprod Contracept Obstet Gynecol 2020;9:4753-5. 